\title{
Haemangioblastomas of the central nervous system in von Hippel Lindau Syndrome involving cerebellum and spinal cord
}

V.A. Kiran Kumar, Vissa Shanti, Yaswanth Sandeep, Jatinder Kumar Mittal, Amit Agrawal INDIA 


\title{
Haemangioblastomas of the central nervous system in von Hippel Lindau Syndrome involving cerebellum and spinal cord
}

\author{
V.A. Kiran Kumar ${ }^{1}$, Vissa Shanti², Yaswanth Sandeep ${ }^{1}$, Jatinder \\ Kumar Mittal ${ }^{1}$, Amit Agrawal ${ }^{1}$ \\ Narayana Medical College Hospital, Chinthareddypalem, Nellore, Andhra Pradesh, INDIA \\ ${ }^{1}$ Department of Neurosurgery; ${ }^{2}$ Department of Pathology
}

Key words: Cerebellar hemangioblastoma, spinal hemangioblastoma, Von HippelLindau Disease

Von Hippel-Lindau (VHL) disease is a multisystem familial syndrome of autosomal dominant inheritance characterized by retinal, cerebellum and spinal cord hemangioblastomas, renal cell carcinomas, adrenal pheochromocytomas, angiomatous or cystic lesions of the kidneys, pancreas, and epididymis. (1) A 41 year old female patient presented with headache from 2 years, one episode of seizure generalised tonic clonic type, two episodes of vomiting. On examination cerebellar signs were positive on right side. MRI Brain (plain \& contrast) was suggestive of well-defined non enhancing hypodense lesion of size $41 \times 28 \mathrm{~mm}$, with eccentric enhancing nodule seen in right cerebellar hemisphere with mild perilesional edema and the lesion is causing mass effect with the shift of cerebellar vermis \& 4th ventricle to the left side s/o right cerebellar hemangioblastoma (Figure 1) She underwent right suboccipital craniectomy and excision of the tumour) (Figure 2). As the patient also had neck pain and paresthesias in both upper and lower limbs and urgency of micturition, patient was evaluated with MRI cervical spine (Plain and contrast) (Figure 3) which was suggestive of heterogenous cystic lesion extending with in central canal from C5-D4 level,with intense heterogenous enhancement noted involving solid portion of lesion at C7\&D1 segments. USG abdomen was s/o Multiple pancreatic cysts, one in tail region $8.6 \times 7.1 \mathrm{~cm}$, polycystic kidney disease, a cystic lesion in right adrenal gland of size $4.1 \times 2.8 \mathrm{~cm}$ and the lesion showing internal vascularity on Colour Doppler flow imaging. Opthalmologic examination visual acuity was 6/9 in both eyes; the intraocular pressure was within normal limits, did not reveal any retinal angiomas. This patient underwent laminectomy and excision biopsy of the lesion. (Figure 4) 
Histopathological examination (HPE) showed anastomosing network of capillary vessels interspersed with nests of stromal cells with moderate amount of pale pink cytoplasm suggestive of haemangioblastoma (Figure 5).

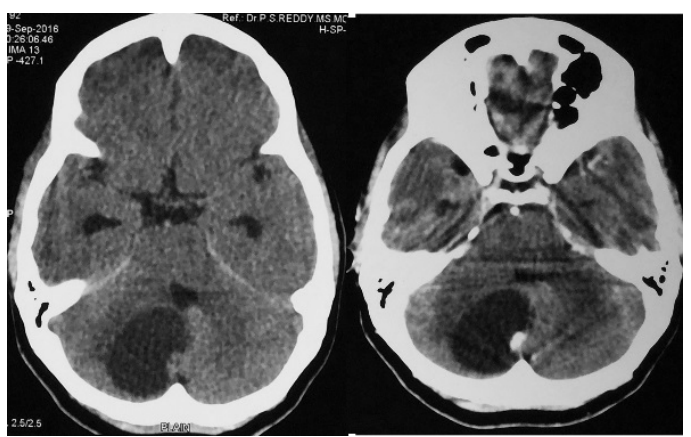

Figure 1 - CT scan of the brain plain and contrast images showing cystic lesion with enhancing nodule in the left cerebellar hemisphere

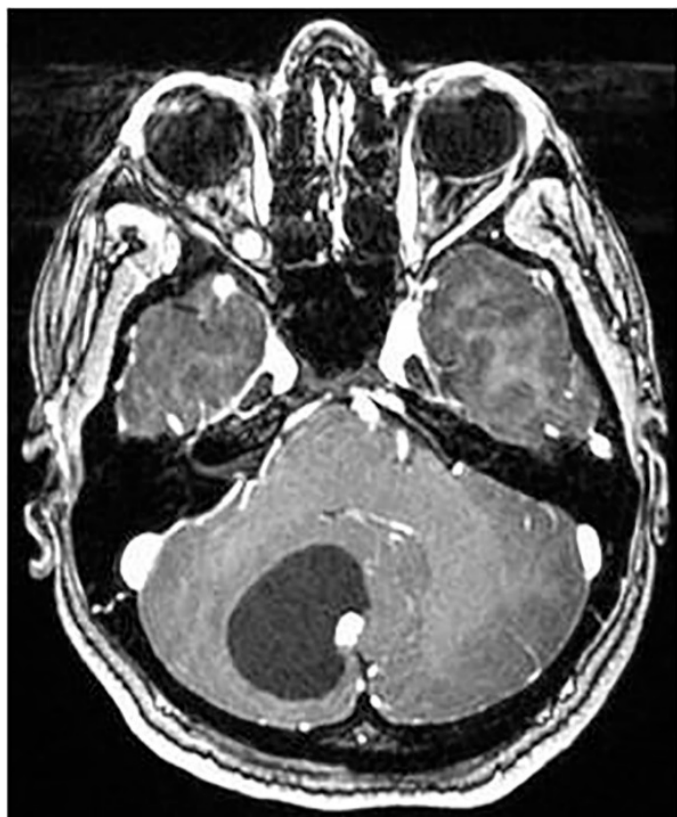

Figure 2 - MRI of the brain contrast image showing cystic lesion with enhancing nodule in the left cerebellar hemisphere

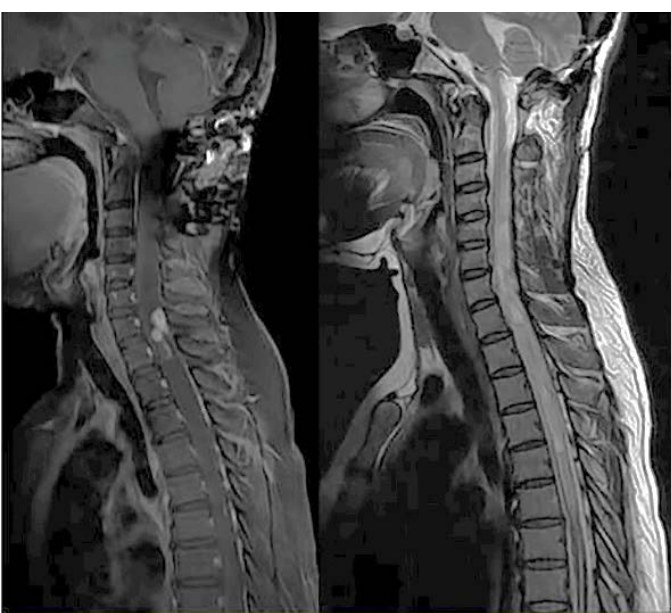

Figure 3 - MRI of the brain contrast image showing cystic lesion with enhancing nodule in the left cerebellar hemisphere and cervical spinal cord

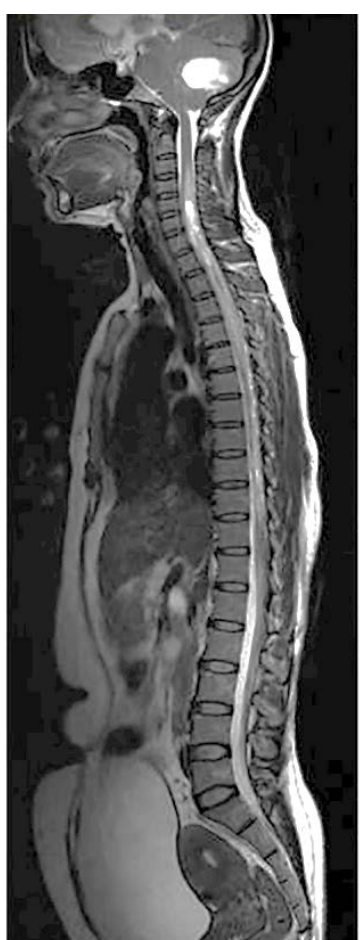

Figure 4 - MRI of the brain contrast image showing cystic lesion with enhancing nodule in the left cerebellar hemisphere and cervical spinal cord 


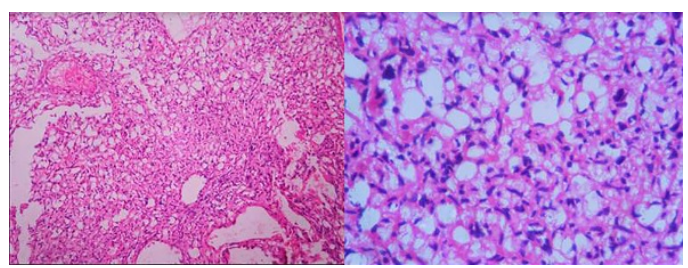

Figure 5 (a and b) - (A) Vascular tumor with tumor cells having vacuolated cytoplasm and hyperchromatic nuclei (H\&E, X100), and (b) the tumour was composed of anastomosing capillary network with interspersed vacuolated stromal cells (H\&E; 400x)

The prevalence of VHL has been estimated to be between 1:35,000-1:40,000. $(2,3) \mathrm{A}$ family history of retinal or central nervous system hemangioblastoma $(\mathrm{Hb})$, only one $\mathrm{Hb}$ or visceral lesion (renal tumours,pancreatic cysts or tumours, pheochromocytoma, papillary cystadenomas oftheepididymis) is required to make the diagnosis of VHL. (4) For isolated cases without a clear family history, two or more $\mathrm{Hb}$ or one $\mathrm{Hb}$ and a visceral manifestation is required. (5) Although hemangioblastomas commonly involve cerebellum, spine and medulla; however when the lesions are multiple the outcome is poorer. (5) As it is obvious the clinical features depend on the location and multiplicity of the lesions. Midline lesions of the cerebellum cause truncal ataxia, laterally situated tumour lesions commonly present with dysmetria. (6-8) The imaging technique of choice for hemangioblastomas is contrast enhanced MRI of the brain and spinal cord. This will show the characteristic lesions cystic lesions with a solid enhancing mural nodule. (9) The definitive treatment of these lesions is complete surgical removal of symptomatic lesions including removal of the mural nodule. $(10,11)$ Gamma
Knife radio surgery has been reported as effective against the solitary small or medium sized mural nodule of haemangioblastoma while the cystic component requires repeated evacuation. (12) Needless to say that the management of hemangioblastomas is more challenging for patients with VHL syndrome as there is presence of multifocal tumour which require high index of suspicion and multiple surgeries.

\section{Correspondence}

Dr Amit Agrawal

Professor of Neurosurgery

Department of Neurosurgery

Narayana Medical College Hospital

Chinthareddypalem

Nellore-524003

Andhra Pradesh (India)

Email-dramitagrawal@gmail.com

dramit_in@yahoo.com

Mobile- +91-8096410032

\section{References}

1.Hamid DA, Abdullah J, Ariff AR, Muhamad M, Madhavan M. Cerebellar hemangioblastoma in a patient with von Hippel-Lindau disease: A case report. The Malaysian journal of medical sciences: MJMS 2000;7:43. 2.Maher ER, Iselius L, Yates JR, et al. Von Hippel-Lindau disease: a genetic study. Journal of medical genetics 1991;28:443-447.

3.Neumann HPH, Wiestler OD. Clustering of features of von Hippel-Lindau syndrome: evidence for a complex genetic locus. The Lancet 1991;337:1052-1054.

4.Memon KL, Rosen SW. Lindau's disease. Am J Med 1964;36:595-617.

5.Bhargava S, Rao A, Mishra A. A Case of Von Hippel Lindau Disease. Med J Armed Forces India 2007;63:302304.

6.Gumprecht H, Lumenta CB. Multifocal hemangioblastoma in a young woman with Hippel- 
Lindau syndrome. Zentralblatt fur Neurochirurgie 1997;59:181-184.

7.Richard S, Martin S, David P, Decq P. Von HippelLindau disease and central nervous system hemangioblastoma. Progress in genetics and clinical management. Neuro-Chirurgie 1998;44:258-266.

8.Weinberg RA. Tumor suppressor genes. Science 1991;254:1138-1146.

9.Quadery FA, Okamoto K. Diffusion-weighted MRI of haemangioblastomas and other cerebellar tumours. Neuroradiology 2003;45:212-219.
10.Lonser RR, Heiss JD, Oldfield EH. Tumor devascularization by intratumoral ethanol injection during surgery. J Neurosurg 1998;88:923-924.

11.Salomé F, Colombeau P, Fermeaux V, et al. Renal lesions in von Hippel-Lindau disease: the benign, the malignant, the unknown. European urology 1998;34:383392.

12.Niemelä M, Lim YJ, Söderman $M$, Jääskeläinen J, Lindquist C. Gamma knife radiosurgery in 11 hemangioblastomas. J Neurosurg 1996;85:591-596. 